\title{
LUCERNAS ROMANAS INÉDITAS DEL MUSEO DE SANTA CRUZ (TOLEDO)
}

\author{
M. MAR ZARZALEJOS PRIETO \\ Dpto. de Prehistoria y Arqueología U.A.M.
}

\begin{abstract}
Abordamos en este trabajo el estudio de un conjunto de lucernas romanas descontextualizadas, pertenecientes a los fondos del Museo de Santa Cruz (Toledo). Se trata de un lote heterogéneo desde el punto de vista tipológico y temporal, cuyo análisis permite adicionar algunas cuestiones que habrán de perfilarse a medida que se produzca el avance de los estudios arqueológicos que se llevan a cabo en diversos puntos de la ciudad de Toledo y su provincia.

On these pages, it is analysed a group of roman lamps without archaeological context from Santa Cruz Museum (Toledo, Spain). This lot presents lamps of type and cronology diferents. Through this annalysis is possible to amount some premiss to know the Roman past in Toledo city and province.
\end{abstract}

En los últimos tiempos hemos venido centrando nuestra atención sobre ciertos conjuntos materiales de época romana albergados por Instituciones museísticas y Colecciones privadas de las Comunidades autónomas de Castilla-La Mancha y Madrid (FERNANDEZ OCHOA-ZARZALEJOSSELDAS, 1989; ZARZALEJOS-AURRECOECHEA-FERNANDEZ OCHOA, 1988; AURRECOECHEA-ZARZALEJOS, 1991; FERNANDEZ OCHOA-ZARZALEJOS, 1991 b). Todos ellos presentan un rasgo común, que no es otro que el de corresponder a hallazgos desprovistos de contexto arqueológico e incluso de procedencia conocida en otras ocasiones. El marco de actuaciones que ha per- mitido el análisis de estos objetos, se inscribe en un proyecto de investigación subvencionado por la U.A.M. y dirigido por la Dra. Carmen Fernández Ochoa durante 1987-88.

El interés por este tipo de estudio, en un momento en el que nuestra disciplina parece decantarse en mayor medida por análisis más globalizadores, responde a la necesidad de clarificar un panorama que, en nuestra opinión, dista de haber sido resuelto. Tal es el caso de numerosos grupos de material, afectados tradicionalmente por lagunas y vacíos de documentación en los mapas de dispersión peninsulares, y cuya existencia está siendo oportunamente probada e interpretada por trabajos recientes. 

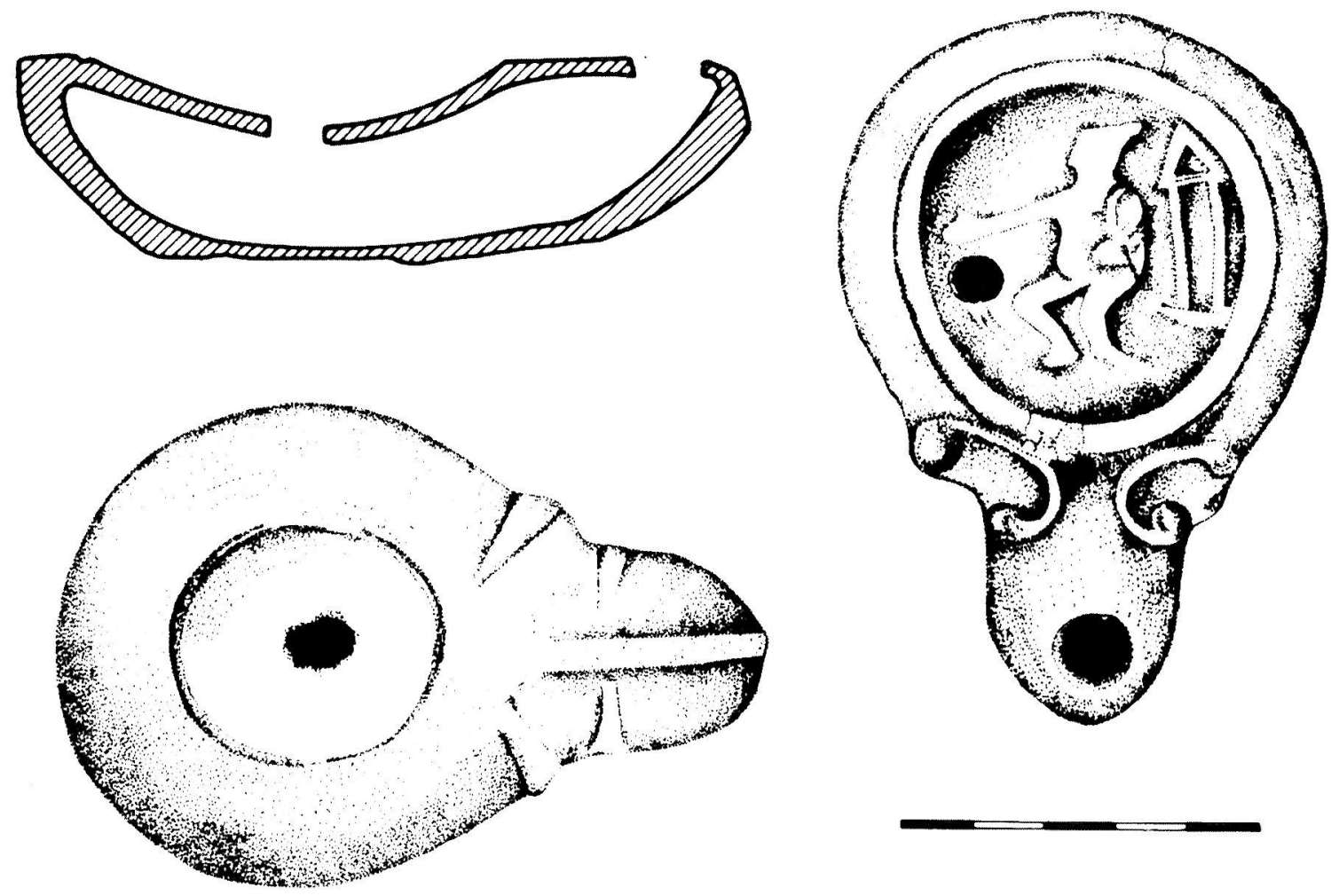

Figura 1.1

En esta ocasión hemos seleccionado un lote integrado por doce lucernas romanas de época muy diversa pertenecientes a fondos del Museo de Santa Cruz (Toledo)! Todas ellas carecen de indicación estratigráfica conocida y pertenecen a hallazgos localizados en diversos puntos, conocidos o no, de la provincia y la ciudad de Toledo. Al margen del interés intrínseco que ofrecen los ejemplares por sus relaciones con otros conjuntos hispanos o foráneos, algunas de las piezas recogidas permiten adicionar datos a la información disponible acerca de la fase tardorromana de Toletum.

Como cabe esperar de una serie como la que presentamos, el lote se caracteriza por su heterogeneidad; por esta razón, y a efectos de facilitar una secuencia lógica, hemos procedido a una ordenación del material establecida en razón de un criterio eminentemente cronológico.

'Deseamos hacer constar nuestró agradecimiento a la Directora del Museo de Santa Cruz, Da . Susana Cortés, que amablemente puso a nuestra disposición las piezas recogidas en este trabajo.

\section{CATÁlogo}

Las piezas objeto de este análisis, han sido clasificadas, como suele ser habitual en materiales de esta índole, a partir de su adscripción tipológica y por ende, de parámetros temporales bien establecidos por otros estudios llevados a cabo fuera y dentro de nuestras fronteras. A este respecto, existe un punto que desearíamos abordar, antes de proceder al comentario individual de las piezas. Ciertamente cualquier estudio sobre lucernas romanas cuenta con la dificultad adicional que le confiere la enorme oferta de cuadros tipológicos brindada por la bibliografía al uso. Esta situación ha llegado a tal grado de complejidad, que puede decirse, sin temor a caer en generalizaciones excesivas, que buena parte de los trabajos centrados en conjuntos numéricamente importantes, proponen su propia tipología, amparada en criterios dispares y no siempre acompañada de las aportunas equivalencias respecto a las sistematizaciones creadas por otros autores. El problema es real aunque no es nuestra intención abundar en cuestiones que han sido abordadas muy recientemente por otro investigador (MORI- 

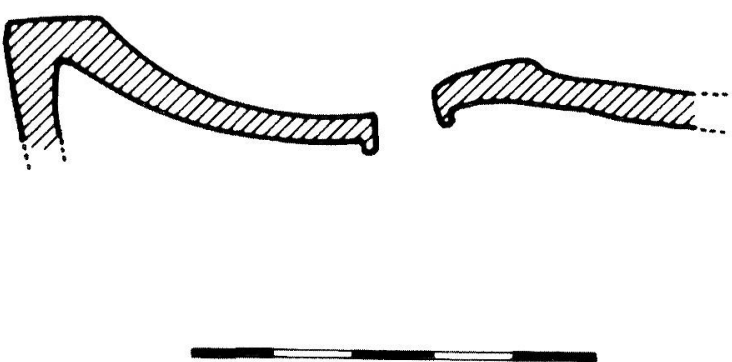

Figura 1.2.

LLO, 1991). Sin embargo, sí hemos creído oportuno aludir a tan espinoso asunto con el fin de aclarar cuál ha sido la opción elegida por nosotros a la hora de asignar una adscripción tipológica para las piezas toledanas. A. Morillo indica en su trabajo la conveniencia de proceder a la adopción de una denominación tipológica unitaria, en la que se especifiquen tan sólo las correspondencias establecidas entre las más completas clasificaciones: Dressel, Loeschcke y Deneauve. Por nuestra parte, compartimos sin reservas la posición de Morillo, dado que la multiplicación de tipos no hace más que complejizar una cuestión que en el fondo se halla satisfactoriamente resuelta desde hace no pocos años. Desde la perspectiva planteada, nos ha parecido oportuno proceder a la clasificación de nuestros ejemplares de época altoimperial de acuerdo con las tres tipologías citadas con anterioridad.

1.- Fig. Núm. 1,1 Inv. 817. Procedencia concreta desconocida. Lucerna completa. Posee una longitud de $100 \mathrm{~mm}$., una anchura máxima de $67 \mathrm{~mm}$. y 26 mm. de altura máxima. Conserva abundantes evidencias de la aplicación de un engobe de coloración castaño-anaranjada de consistencia escasamente uniforme. Se trata de una lucerna de cuerpo circular, con volutas apenas sobresalientes en el perfil y piquera redondeada ligeramente ojival. Presenta margo liso, separado del discus mediante una estrecha moldura. Este último ofrece una superficie de concavidad suave, en la que pueden reconocerse las líneas maes-

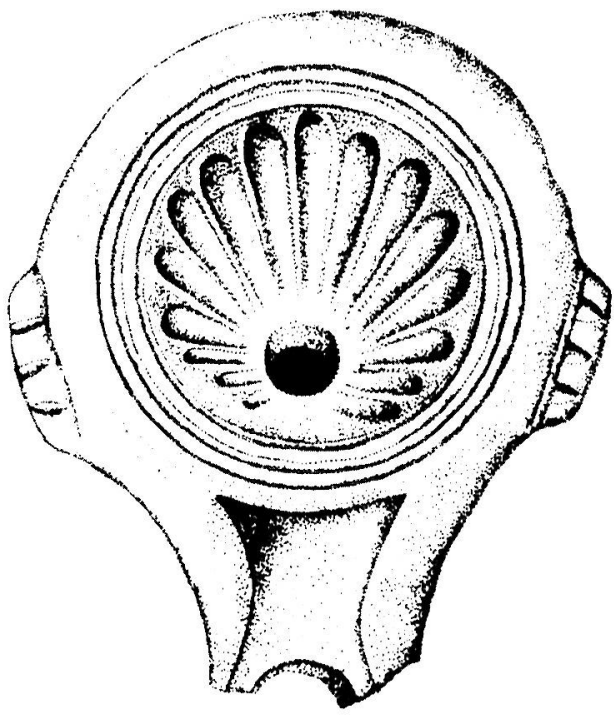

tras de su decoración original pese a encontrarse bastante desgastada. En efecto, resulta visible una figura masculina hacia la izquierda, tocada con pileus, con la pierna derecha flexionada y la izquierda apoyada en el suelo, al tiempo que extiende hacia adelante el brazo y la mano derecha, mientras que con la izquierda parece sujetar el manto. Junto a esta figura y en segundo plano, puede reconocerse la representación sumaria de un edificio provisto de frontón y dos columnas en la fachada. La escena no resulta novedosa en el repertorio de decoraciones sobre lucernas romanas, hasta el punto que ha podido ser identificada como la representación de Ulises (Odysseus) en actitud declamante. Tal es la interpretación ofertada para los paralelos rastreables en el British Museum (WALTERS, 1914, 100, núm. 658; BAILEY, 1980, 176, Q 929, lám. 17), Thamusida y Cotta (PONSICH, 1961, 84, núm. 63, lám. VI 63; 115, núm. 474, lám. XXXI, 474). Idéntica opinión ha sido defendida para los ejemplares hispanos que documentan esta representación, a saber, una pieza de procedencia desconocida depositada en el Museo de Sevilla (FERNANDEZ CHICARRO, 1956, 74, núm. 57, fig. 46, núm. 9) y otra proveniente de Mérida que engrosa asimismo los fondos del Museo de la ciudad (GIL FARRES, 1947-8, 106, lám. III, núm. 21), ambas muy próximas a la que presentamos en cuanto a ejecución y dimensiones.

La presencia de escenas relacionadas con el mito de Ulises en la lucernaria romana de procedencia itálica no resulta comparativamente demasiado frecuen- 
te (BAILEY), 1980, 35). No obstante, son varios los episodios de la Odisea homérica que han sido documentados sobre este tipo de objeto: Ulises y las Sirenas, Ulises y Polyphemus (DAREMBERGSAGLIO, 1986, III.2, 1326), Ulises y Neoptolemus con Philoctetes, etc. (BAILEY, 1980, 35). La escena concreta que hemos reconocido sobre el ejemplar toledano, podría representar a juicio de algunos autores, a Ulises en actitud de declamación, delante de lo que puede interpretarse como la valva hospitalis de la scaena de un teatro (GIL FARRES, 1947-8, 106).

Por lo que se refiere a la adscripción tipológica de nuestra pieza, es de destacar su plena coincidencia con la de los paralelos citados; se trata de una lucerna de volutas con mixus redondeado perteneciente al tipo Dressel 11, Loeschcke IV, Deneauve VA.

La datación global propuesta por buena parte de los autores interesados en esta cuestión, sitúa estas producciones en el siglo I d.C., si bien existen ciertas divergencias en la matización de períodos concretos que conviene anotar. Así parece existir cierto acuerdo en la situación del inicio del tipo en la primera mitad del siglo, posición en la que se integran las propuestas de Loeschcke, Deneauve, Ponsich, Szentlelely, Walters, Bruneau y Bailey entre otros (LOESCHCKE, 1919, 225; DENEAUVE, 1969, 126; PONSICH, 1961, 31; SZENTLELEKY, 1969, 79; WALTERS, 1914, 95-124; BRUNEAU, 1965, 116; BAILEY, 1980, 172). Por lo que respecta al marco temporal en que ha de situarse el fin de esta producción, se detectan dos posturas básicas que abogarían por los últimos años del siglo I (DENAUVE, 1969, 126; BELCHIOR, 1969, 33; PONSICH, 1961, 31) o que prolongan su perduración en los albores del III d.C. (PROVOOST, 1976, 554; SZENTLELEKY, 1969, 79; BAILEY, 1980, 172). Por su parte López Rodríguez considera el tipo en cuestión inserto entre los años 40-70 d.C., mientras que las lámparas de igual forma aunque provistas de asa pudieron perdurar hasta época de Adriano (LOPEZ RODRIGUEZ, 1981, 106).

2.- Fig. 1,2. Núm. Inv. 818. Procedencia concreta desconocida. Lucerna incompleta. Conserva una longitud máxima de $85 \mathrm{~mm}$. y $74 \mathrm{~mm}$. de anchura. Presenta pasta de coloración ocre, con restos blanquecinos en superficie. La fragmentación de la pieza afecta al mixus y al fondo del objeto, del que nada se conserva.

La decoración del discus se halla constituida por una venera de gallones cóncavos en cuya confluencia se sitúa el orificio de alimentación. Disco y margo aparecen separados por dos finas molduras. La pieza, que carece de asa posterior, está dotada de agafes o aletas laterales de forma aproximadamente trapezoidal y provistas de tres incisiones en la superficie anterior. La piquera, hoy perdida, debió presentar remate triangular, con volutas apenas insinuadas tal y como puede reconocerse en el estado actual del objeto.

Los rasgos morfológicos y técnicos documentados por este ejemplar, permiten incluirle en el marco de un conjunto de lucernas bastante homogéneo y bien reconocido en no pocos puntos de la geografía peninsular. Se trataría sin duda, de una pieza fabricada en los talleres de Andújar (SOTOMAYOR et alii, 1976, 132135; SOTOMAYOR et alii, 1979, 489; SOTOMAYOR et alii, 1981, 309-316), tal y como ha podido ser inferido a partir de los hallazgos radicados en este centro productor bético. Desde el punto de vista tipológico, este modelo de lucerna fue clasificada en su día como una derivación del tipo Dressel 3 (Deneauve III) de cronología republicana, aunque desprovista del asa posterior (LOPEZ RODRIGUEZ, 1981, 100; IDEM, 1982, 381). El marco temporal en que han de situarse tales productos ha sido inferido por el último autor citado, de la data de los basureros de Andújar donde han sido localizados, así como de estratigrafías provistas de lucernas de este tipo. De este modo ha podido diseñarse un ámbito cronológico situable entre época julio-claudia y quizá el período flavio (LOPEZ RODRIGUEZ, 1981, 100; IDEM, 1982, 382), aunque ha de tenerse presente que su fabricación comenzó a experimentar un proceso de rarificación desde el período claudio (ROCA, 1980, 270-271).

Una vez admitida la procedencia y datación apuntadas, sin duda uno de los aspectos más interesantes que genera el análisis de estas lucernas, es el que se refiere al área de dispersión conocida hasta el presente. En efecto, parece que la mayor parte de los hallazgos se concentran de modo preferente en el área andaluza, tal y como cabía suponer a juzgar por la habitual presencia en la zona de TSH procedente de los alfares de Andújar. No citaremos pormenorizadamente los lugares de este ámbito espacial en los que han sido reconocidas lucernas de este origen, dado que J.R. López Rodríguez incluye en sus trabajos oportuna referencia a este particular (LOPEZ RODRIGUEZ, 1981, 100; IDEM, 1982, 382). Sin embargo, como ya apuntara este mismo investigador, no deja de resultar curiosa la aparición de ejemplares de procedencia giennense en lugares donde no es nada fre- 
cuente la presencia de TSH fabricada por los talleres de Andújar. Tal es el caso de Almenara de Adaja (Valladolid) (DELIBES-MOURE, 1974, 32, Fig. 14, 1), Calahorra (AMARE, 1984, 139-142; IDEM, 1987, 30 y 50, Lám. I, 2), Puelles (Asturias) (FERNANDEZ OCHOA, 1980, 326, Fig. 3), Conimbriga (BELCHIOR, 1969, núm. 1 y 2), quizá Pontevedra (FARINA, 1976, 10) y Herrera de Pisuerga (Palencia) ${ }^{2}$. Hasta hace algún tiempo, las causas reales atribuibles a un comportamiento como el que reflejamos, podían ponerse en relación con la ausencia de trabajos detenidos, que abordaran en detalle el problema de la comercialización de los centros de producción de TSH en zonas geográficas tan interesantes como la Meseta Sur. Sin embargo, nuevos datos contrastados muy recientemente, permiten abordar la cuestión con mayores elementos de juicio. Seleccionaremos a tal efecto los resultados obtenidos por nosotros mismos en dos áreas que, desde nuestro punto de vista, pueden arrojar alguna luz a este problema. Las primeras puntualizaciones se refieren al estudio de TSH proporcionada por el solar de la antigua Sisapo (La Bienvenida, Almodóvar del Campo, Ciudad Real). Este análisis ha permitido comprobar la marcada preponderancia de las producciones hispánicas fabricadas en Andújar, dato que concuerda perfectamente con el interesante volumen de lucernas de idéntica procedencia (FERNANDEZ OCHOA-SELDAS, 1987, 265-266; FERNANDEZ OCHOA et alii, 1990). Conviene anotar que esta constatación no resulta óbice para la documentación de un conjunto nada desdeñable de origen riojano (FERNANDEZ OCHOA-ZARZALEJOS, 1991a). Idéntico fenómeno de binomio comercial ha sido reconocido en el lote de materiales de la Colección de D. Eduardo Tello albergado por el Museo Provincial de Ciudad Real e integrado por piezas recogidas en yacimientos de esta provincia (FERNANDEZ OCHOA-ZARZALEJOS, 1991 b)

La segunda zona objeto de atención se refiere a diversos puntos de la Comunidad de Madrid y la Provincia de Toledo, donde los resultados obtenidos manifiestan sensible diferencia con los expuestos más arriba. En efecto, la interpretación de algo más de una treintena de marcas de ceramista sobre TSH localizadas en siete yacimientos diferentes, permite contrastar

\footnotetext{
${ }^{2}$ Según comunicación oral de A. Morillo, quien incorpora uno de estos ejemplares en su Memoria de Licenciatura sobre las Lucernas de Herrera de Pisuerga.
}

la ausencia completa de materiales asignables a los alfares giennenses (FERNANDEZ OCHOA-ZARZALEJOS-SELDAS, 1989, 1111). El dato parece estar ratificado por el estudio monográfico aplicado a la T.S. de uno de los yacimientos madrileños considerados en el trabajo anterior: Velilla de San Antonio (ZARZALEJOS, 1990), donde en un conjunto que supera ampliamente los quinientos fragmentos, ni uno sólo ha podido ser identificado como perteneciente a los talleres béticos.

Así las cosas, resultaría inferible una primera conclusión parcial que certifica lo que hace algunos años parecía probable y que hoy resulta confirmado en mayor medida. Tal verificación, apuntaría hacia una escasísima distribución de la TSH producida en Andújar al N. del Guadiana, como bien demuestran los trabajos efectuados sobre yacimientos tan relevantes como Numancia (ROMERO, 1985), Herrera de Pisuerga (PÉREZ GONZALEZ, 1989), Complutum (FERNANDEZ GALIANO, 1984) o la propia Segóbriga y las ciudades de su entorno contempladas recientemente (SANCHEZ-LAFUENTE, 1990, 380). No obstante, conviene matizar una vez más que tal panorama puede resultar modificado mediante la publicación de nuevos conjuntos. A este respecto nos resulta interesante citar una mención sobre la presencia de T.S.H. procedente de Andújar en Coca (Segovia), si bien no existe hasta el momento una publicación pormenorizada del material, que certifique la presunción de su origen, a través de marcas de alfarero, punzones idénticos o cualquier otro elemento de juicio que permita afirmar con seguridad su procedencia (BLANCO GARCIA, 1988, 55; IDEM, 1987, 27).

En cuanto al problema que nos atañe, hemos de indicar que no contamos hasta el presente, con argumentaciones definitivas que autoricen la explicación razonada de este fenómeno. Podría apuntarse a modo de hipótesis, la posibilidad de que los canales de distribución de las lucernas y la terra sigillata de Andújar se mantuvieran diversificadas en cuanto a gestión. Sin embargo, parece más probable que se tratara de una simple cuestión de demanda y por ende, de falta de competencia con los potentes centros del valle del Ebro, que no comenzaron a fabricar utensilios de este tipo hasta época más tardía, al menos según los últimos datos conocidos al respecto (SOLOVERAGARABITO, 1986, 119 y 121; GARABITO et alii, 1986, 135; SOLOVERA-GARABITO, 1990, 76). 

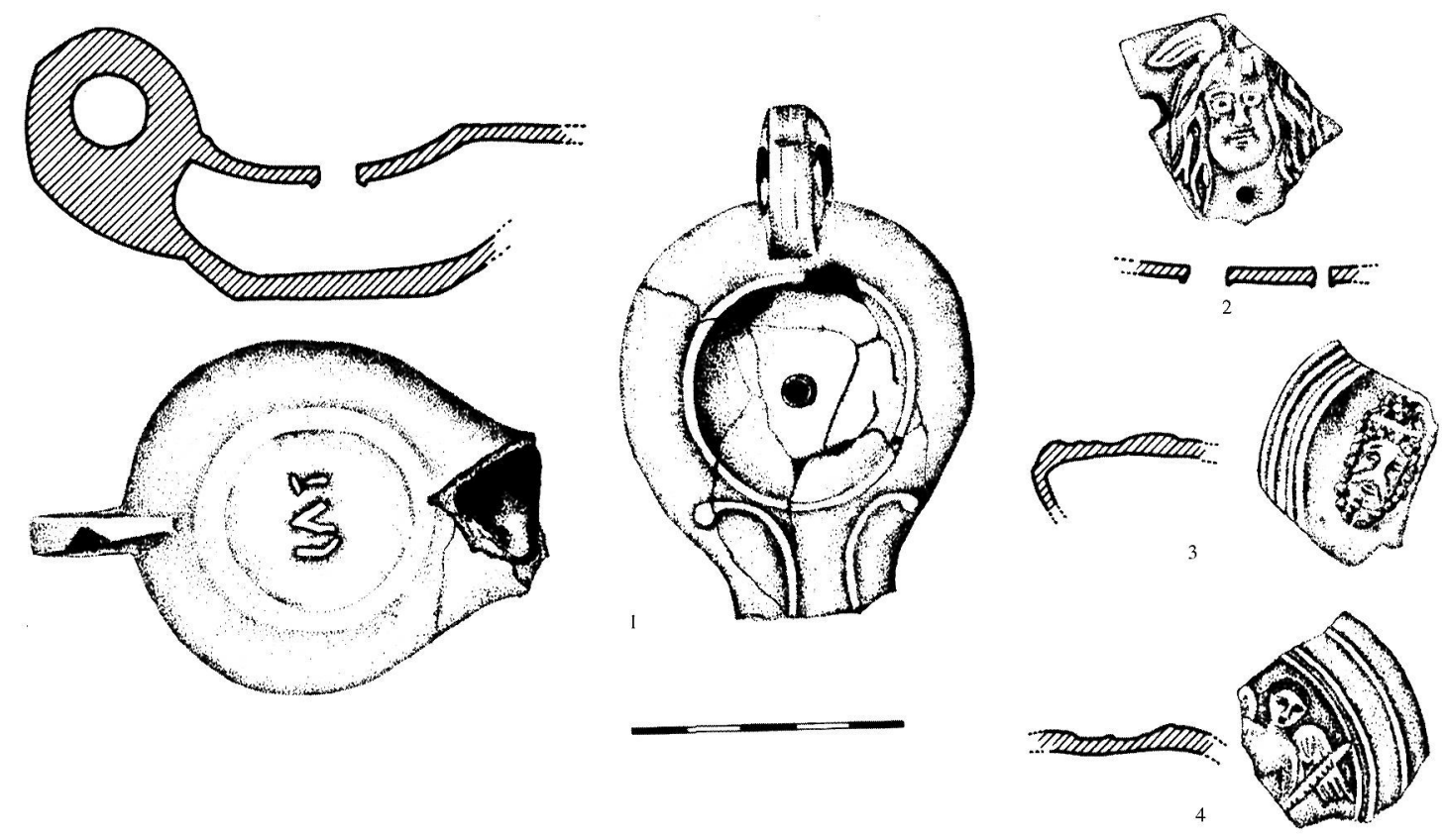

Figura 2

3.- Fig. 2,1. Núm. Inv. 526. Procedencia concreta desconocida. Lucerna incompleta. Posee $65 \mathrm{~mm}$. de anchura máxima, una altura de $51 \mathrm{~mm}$. desde la base hasta la zona superior del asa y $77 \mathrm{~mm}$. de longitud máxima conservada. Presenta pasta de coloración anaranjada y restos de un engobe superficial de tonalidad crema-ocre. Ostenta cuerpo circular, provisto de discus de concavidad suave con orificio de alimentación centrado, margo liso y asa posterior perforada. La pieza en cuestión, que ha perdido el mixus, conserva evidencias de haber poseído volutas no sobresalientes en el perfil del objeto. La base del ejemplar ostenta una marca de alfarero inscrita en un círculo: LAT.

Los rasgos técnicos y morfológicos descritos, así como la marca identificada permiten la incorporación de esta pieza en el marco de una derivación bien establecida de la forma Dressel 9 (Loeschcke I, Deneauve IV A), más conocida en la bibliografía nacional como «lucerna minera» (LUZON, 1967, 138-150). Tal denominación se halla relacionada con los lugares donde preferentemente han sido localizadas: enclaves de rancia tradición minera o necrópolis relacionadas con habitats cuya actividad económica se hallaba orientada a la explotación de minerales (LUZON, 1967, 138-9; LOPEZ RODRIGUEZ, 1981, 104). El número de hallazgos es importante tal y como puede comprobarse a través de la relación ofertada por los trabajos citados. Nos parece oportuno destacar sin embargo, la presencia de un nuevo ejemplar publicado con posterioridad a los anteriores en Sisapo (La Bienvenida, Ciudad Real), cabeza de un importante distrito minero (FERNANDEZ OCHOA-SELDA, 1987, 269, núm. 51, Fig. 15,H).

El ejemplar toledano que presentamos, puede ser identificado con el primero de los grupos (A), establecido por J.M. Luzón en el que se incorporan las lámparas de factura más cuidada, datables según este mismo autor en torno al cambio de centuria (LUZON, 1967, 141).

Desde otro punto de vista, J.R. López Rodríguez ha establecido una nueva clasificación, en la que contempla ejemplares con margo decorado y aquellos que lo presentan liso como en el caso que ofrecemos (LOPEZ RODRIGUEZ, 1981, 104). Este investigador intuye cierta evolución cronológica entre ambos modelos, aunque, como muy bien reconoce, sin argumentos que lo certifiquen, de tal modo que las lámparas lisas pudieran ser posteriores a las decoradas (LOPEZ RODRIGUEZ, 1981, 104). A nuestro juicio tal distinción no puede ser planteada en términos absolutos, al menos si se tiene en cuenta un conjunto tan homogéneo como el de Riotinto. En efecto, partiendo 
de la diferenciación amparada en el acabado establecida por Luzón, se advierte que existe un importante número de piezas decoradas que, en virtud de su calidad de ejecución, fueron incluidas por este último autor en la categoría B, integrada por lucernas de apariencia más tosca e insertable de modo genérico en la mitad del siglo II d.C. (LUZON, 1967, 141-143).

Por lo que respecta a la marca de alfarero, ha de anotarse su documentación en el ya citado conjunto de Riotinto en una lámpara de características muy similares a la nuestra (LUZON, 1967, 145, Fig. 8-9, núm. 43), así como en otra lucerna minera de procedencia portuguesa (FERREIRA ALMEIDA, 1953, Núm. 123)

Algo se ha esbozado en líneas anteriores acerca de la cronología aplicable a este tipo de lámpara. El estudio de las asociaciones con otros materiales bien fechados en diversos yacimientos, ha permitido a López Rodríguez establecer un amplio marco temporal situable durante el siglo II d.C. y una parte nada desdeñable del siglo III (LOPEZ RODRIGUEZ, 1981, 104).

Un último punto a considerar en torno a esta pieza es el que se refiere a su posible procedencia. Ciertamente los datos de que dispone el Museo nada permiten aventurar acerca de un origen concreto. Sin embargo, la relación evidente entre este tipo de lucernas y los yacimientos mineros induce a buscar posibilidades de esta índole en el marco de la provincia de Toledo, admitiendo que el ejemplar provenga, como parece, de algún punto de este ámbito territorial. En este sentido, el conocimiento de la existencia de explotaciones metalíferas antiguas no resulta aquí demasiado prolijo. Domergue ha podido localizar dos zonas de cierto interés: Santa Quiteria (Sevilleja de la Jara) y Nava de Ricomalillo (DOMERGUE, 1987, 485-487). La primera de ellas, situada en el corazón de los Montes de Toledo, documenta dos yacimientos de plomo argentífero que proporcionaron «lámparas de tierra cocida», así como material propio de una explotación antigua de este género cuyo paradero se desconoce (DOMERGUE, 1987, 485).

El área minera de Nava de Ricomalillo se halla emplazada en el reborde N. de los Montes de Toledo. Los análisis realizados sobre esta zona permiten argumentar la explotación romana de filones auríferos en relación con un habitat de esta misma adscripción cultural en lugar muy próximo (DOMERGUE, 1987, 486-87).

A tales áreas podría sumarse una tercera reconocida por Caballero Zoreda y Sánchez Palencia en las inmediaciones de Melque (CABALLEROSANCHEZ PALENCIA, 1982, 392).

Ciertamente tales datos únicamente resultan eficaces desde el punto de vista ilustrativo, puesto que nada sabemos del período concreto durante el que se mantuvo la actividad extractiva en estos puntos. En otras palabras, permiten reconocer la existencia de explotaciones mineras romanas en la provincia de Toledo, a las que hipotéticamente pudieran estar asociadas lucernas del tipo que nos ocupan.

4.- Fig. 2,2. Núm. Inv. 875. Procedencia concreta desconocida. Fragmento de disco de lucerna de forma indeterminada. Presenta pasta de coloración beige crema y superficie recubierta por un engobe de tonalidad castaña. La porción de discus conservada permite reconocer una cabeza de Medusa provista de alas. Este género de representación registró cierta frecuencia en el campo de la lucernaria romana (LOPEZ RODRIGUEZ, 1981, núm. 163; RODRIGUEZ NEILA, 1977, 387, núm. 7; QUINTERO ATAURI, 1932, núm. 117, lám. XII; LOESCHCKE, 1919, Lám. VII, núm. 70 y 392) así como en otros ámbitos de las artes romanas (GARCIA MERINO, 1990, 116, núm. 130). Una representación del mismo género, aunque con las alas situadas en el cuello ha sido reconocida en Segóbriga (ABASCAL, 1989, 315, núm. 70, Fig. 7). Por su parte, el British aloja en sus fondos un ejemplar con el mismo tipo de figuración aunque de estilo bastante distinto a la que nos ocupa (BAILEY, 1980, 312, Q 1245). Finalmente destacamos las conexiones estilísticas existentes entre la representación del Museo de Santa Cruz y la documentada sobre una lucerna de disco procedente de Cotta, interpretada en su caso como cabeza de mujer (PONSICH, 1961, 103, Lám. XXIII, 319).

Por lo que respecta a la datación de la pieza, carecemos obviamente de datos relacionables con la tipología de lucerna a la que perteneció el fragmento en cuestión. Tampoco el tema decorativo parece aportar argumentos reveladores, dado que si bien las representaciones de Medusa suelen ser frecuentes en el siglo I d.C. (LOPEZ RODRIGUEZ, 1981, 119) no faltan ejemplares encuadrables en la siguiente centuria (BAILEY, 1980, 312).

5.- Fig. 2,3. Núm. Inv. 877. Procedencia concreta desconocida. Fragmento de disco de lucerna de forma indeterminada. Presenta pasta de coloración beige claro y superficie cubierta por un engobe lige- 
ramente anaranjado. Conserva parte del discus, decorado con una cabeza masculina barbada de frente, así como el arranque de la pared del infundibulum. No hemos hallado paralelos idénticos para la representación, aunque podría tratarse de una máscara teatral.

La ubicación temporal de la pieza presenta la misma problemática que hemos anotado en el caso anterior: la imposibilidad de adjudicar el fragmento a un tipo concreto de lámpara.

6.- Fig. 2,4 Núm. Inv. 876. Procedencia desconocida. Fragmento de disco de lucerna de forma indeterminada. Presenta pasta de coloración crema y superficie con restos de engobe castaño rojizo mal conservado. Pese a la exigüidad del fragmento, puede reconocerse parte de la ornamentación del discus, en la que resulta visible una figura infantil alada, interpretable como Eros o Cupido en actitud no identificada.

7.- Fig. 3. Núm. Inv. 527. Procedencia concreta desconocida. Lucerna incompleta. Conserva $115 \mathrm{~mm}$. de longitud máxima, $80 \mathrm{~mm}$. de anchura y $30 \mathrm{~mm}$. de altura máxima. Presenta pasta anaranjada de cocción poco uniforme y superficie castaño-anaranjada con huellas de uso en la zona conservada de la piquera. Se trata de una lucerna de cuerpo circular, con asa horadada y discus de concavidad suave, desprovisto de decoración, con orificio de alimentación centrado así como otro para ventilación situado en la línea del eje de la piquera. El margo, separado del disco mediante una moldura, se halla ornamentado por doble hilera de perlas en relieve. No conserva el mixus completo, aunque parece evidente que responde al tipo redondeado.

Buena parte de las características apuntadas incorporan este ejemplar en el grupo conocido como «lucernas de disco», correspondientes a los tipos Dressel 17, 18, 19, 20, 24, 25 y 27, aunque conviene hacer notar que la ausencia en este caso, de cualquier elemento de separación entre el margo y la piquera impide asignarlo a una variante concreta de las enunciadas por Dressel, aunque el tipo sí es recogido por Deneauve como VIII C (DENEAUVE, 1969, 81). Además de los ejemplares procedentes de Cartago (DENEAUVE, 1969, núm. 1012-1021), este rasgo, aunque no muy frecuente en los conjuntos de este tipo, ha podido ser documentado en un ejemplar completamente liso de procedencia desconocida, perteneciente
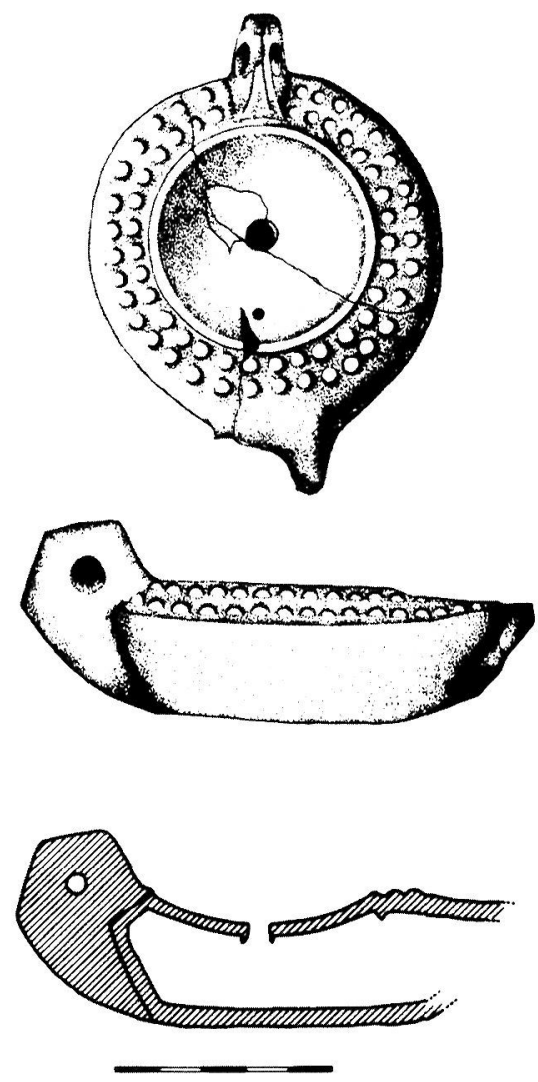

Figura 3

al Museo de Historia húngaro (SENTLELEKY, 1969, núm. 161), así como en Conimbriga, donde se hace notar la escasa presencia de esta modalidad y donde se registra una pieza con el mismo tipo de decoración que la que presentamos (MOUTINHO ALARÇAO-DA PONTE, 1976, 99, Lám. XXVI, Núm. 66).

Parece que la producción de esta tipología genérica de lucernas puede arrancar del período claudioneroniano (PONSICH, 1961, 334; SZENTLELEKY, 1969, 101; PAVOLINI, 1977, 38) prolongándose durante el siglo II e incluso el III para las variantes cordiformes (PONSICH, 1961, 35).

Por lo que respecta al tipo de decoración documentado en este ejemplar toledano, ha de apuntarse que su presencia en otras variantes de lucernas de disco no resulta infrecuente en ningún caso (PONSICH, 1961, lám. XVII, núm. 210; lám. XVIII, núm. 234), aunque curiosamente parece prodigarse en mayor medida sobre los modelos acorazonados, que como se ha indicado con anterioridad pueden ser los más tardíos (BAILEY, 1980, Lám. 86, Q 1421 y 1422; 

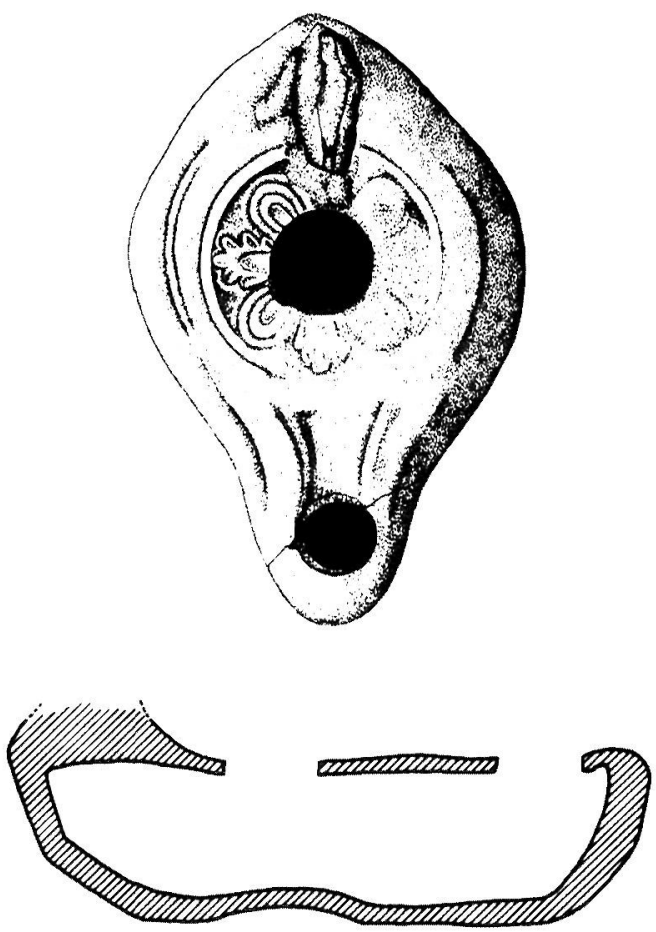

Figura 4

SZENTLELEKY, 1969, núm. 146; PONSICH, 1961, Lám. XXIV, núm. 327; MOUTINHO ALARÇAODA PONTE, 1976, Lám. XXVI, núm. 64; MANERA, 1960, Lám. XXXII núm. 1 y 9).

Las dimensiones de nuestro ejemplar, la sección del asa, que evoluciona hacia modelos triangulares documentados también en el siglo III (PONSICH, 1961, 43), unido a la tardía datación proporcionada por el fragmento de Conimbriga citado más arriba (MOTINHO ALARÇAO-DA PONTE, 1976, 99), permitiría situar en época avanzada esta modalidad de lucerna. Más aún, el tipo de decoración que estamos tratando, aparecerá sobre las lámparas de forma Dressel 30 o Loeschcke VIII tardía con el margo ornamentado con puntos o perlas en relieve (Warzenlampen), datables desde fines del siglo III hasta comienzos del $\mathrm{V}$, y que no pocos autores interpretan como la última evolución de las lucernas de disco (LOPEZ RODRIGUEZ, 1981, 116). Es posible, a nuestro juicio, que el supuesto modelo a partir del cual se produce esta evolución tuviera bastante que ver con lámparas de características muy similares a las que documenta nuestro ejemplar. Esta apreciación podría venir avalada por diversos rasgos de índole formal, entre los que destacan la presencia de disco liso rodeado por una moldura, cuerpo de importantes dimensiones, y decoración de perlas en el margo.

8.- Fig.4. Num. Inv. 208. Procedencia: Moraleda (Toledo). Lucerna completa. Presenta una longitud máxima de $125 \mathrm{~mm}$., $78 \mathrm{~mm}$. de anchura y una altura máxima de $34 \mathrm{~mm}$. sin tener en cuenta el elemento de prensión, que se halla fragmentado en su base. Documenta pasta de tonalidad ocre crema y superficie de coloración beige, enmascarada por la presencia de abundantes concreciones. Conserva huellas de utilización en la piquera. Ofrece cuerpo ovoide, con disco plano provisto de un orificio de alimentación centrado de diámetro importante $(20 \mathrm{~mm}$., en torno al cual se desarrolla el tema floral que sirve de ornato al disco. Este último se halla conectado con el mixus a través de un canal. El margo aparece profusamente afectado por las concreciones a que hicimos referencia en la descripción, circunstancia ésta que no resulta óbice para estimar la ausencia de cualquier tipo de decoración en relieve o impresa.

Este ejemplar puede ser inscrito desde el punto de vista tipológico y por ende, temporal, en el grupo de lucernas conocidas como «africanas» (Afrikanische Lampen) o «paleocristianas». Tales denominaciones hacen referencia respectivamente, bien al emplazamiento geográfico de sus centros de producción (MENZEL, 1969, 90), bien a su prolija documentación en catacumbas cristianas o a los motivos ligados a esta simbología que las decoraron. Ambos términos son aceptados por la bibliografía al uso, pese a que se admite la impropiedad inherente a cualquiera de las dos denominaciones (DE CAROLIS, 1988, 22; SZENTLELEKY, 1969, 127).

Dentro de las sistematizaciones más difundidas, tales lámparas corresponden a los tipos Dressel 31, Hayes I B, Provoost 6, Pohl 2, Ponsich IV B, Broneer XXXI, Leibundgut XXXVII, Atlante IX c, entre otros.

La decoración presente en el ejemplar toledano, consiste en un motivo floral, reconocible sólo en parte y que parece estar constituido por una roseta en la que alternan pétalos sencillos y lobulados. No ha sido posible documentar idéntica formación sobre otros ejemplares publicados, aunque la aplicación de esquemas decorativos de concepción similar resultó frecuente en producciones de estas características (HOFF, 1987, 99-100, núm. 35-37; PONSICH, 1961, 

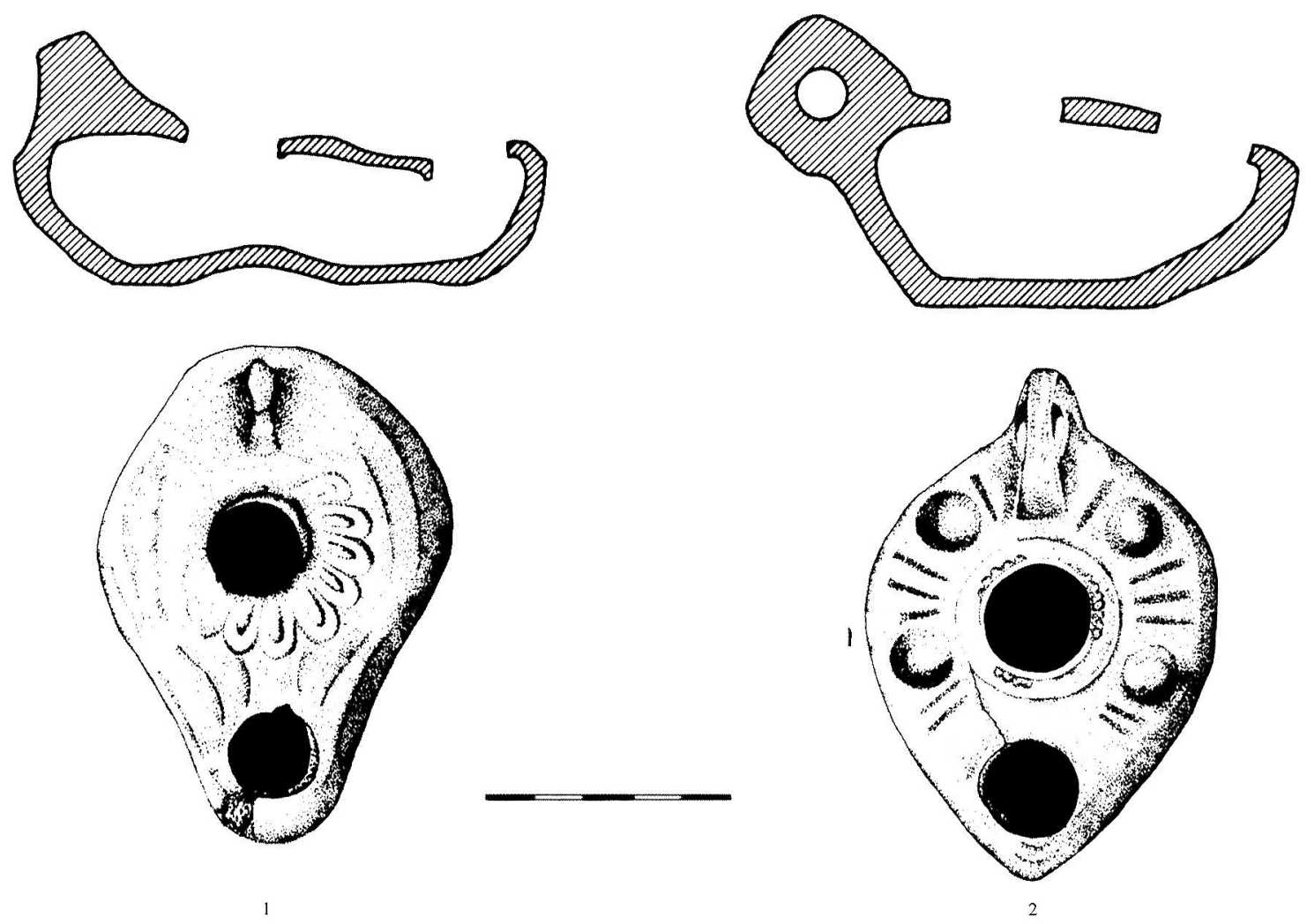

Figura 5.

Lám. XXVI, núm. 358; GIL FARRES, 1947-48, 111 , núm. 45; AMARE, 1987, 31, Lám. III, 10).

Acerca del origen de este tipo de lámpara, buena parte de los especialistas interesados en la cuestión sitúan los focos de producción en diversos puntos de la geografía norteafricana; en este sentido, por citar algunos ejemplos, se pronuncia Provoost (PROVOOST, 1970, 27-29; 44-47), o Pohl, Szentléleky y Michelucci quienes se suman a la hipótesis de su origen alejandrino o de modo más genérico egipcio (POHL, 1962, 225-7; SZENTLELEKY, 1969, 127; MICHELUCCI, 1975, 97), mientras que Hayes considera su tipo I como de fabricación tunecina (HAYES, 1972, 313). No obstante, la importante difusión de estos modelos en el Mediterráneo occidental, debió favorecer el surgimiento de imitaciones locales (BAILEY, 1980, 382; DE CAROLIS, 1988, 22), algunas de las cuales han sido detectadas en la propia Italia (BAILEY, 1980, 383-384) así como en algún yacimiento hispano, como Lucentum (Benalúa, Alicante) (REYNOLDS, 1987, 133). En el caso que nos ocupa, es conveniente advertir tanto la ausencia de decora- ción en el borde o margo, elemento éste que marca la diferencia con los productos correspondientes al tipo Hayes I (HAYES, 1972, 310-314), como las características de pasta y superficie, bien alejadas de los prototipos de Terra Sigillata Clara C (African Red Slip Ware).

Finalmente, por cuanto se refiere al marco temporal aplicable a lucernas de este género, Hayes sitúa su tipo I en el siglo IV y primeros años del V d.C. (HAYES, 1972, 313), cronología ésta a la que se ciñe buena parte de los repertorios que venimos citando.

9.- Fig. 5,1.- Inv. 209. Procedencia concreta desconocida. Lucerna completa. Cuenta con $102 \mathrm{~mm}$. de longitud máxima, $72 \mathrm{~mm}$. de anchura y una altura de $52 \mathrm{~mm}$. desde el elemento de prehensión hasta la base. Documenta superficie de coloración crema, con indicios de utilización en la piquera. Presenta cuerpo de forma ovoide algo irregular, con discus menos individualizado que en el ejemplar precedente. En su centro, ofrece un orificio de alimentación rodeado por un motivo floral, en parte muy desdibujado. La unión con 

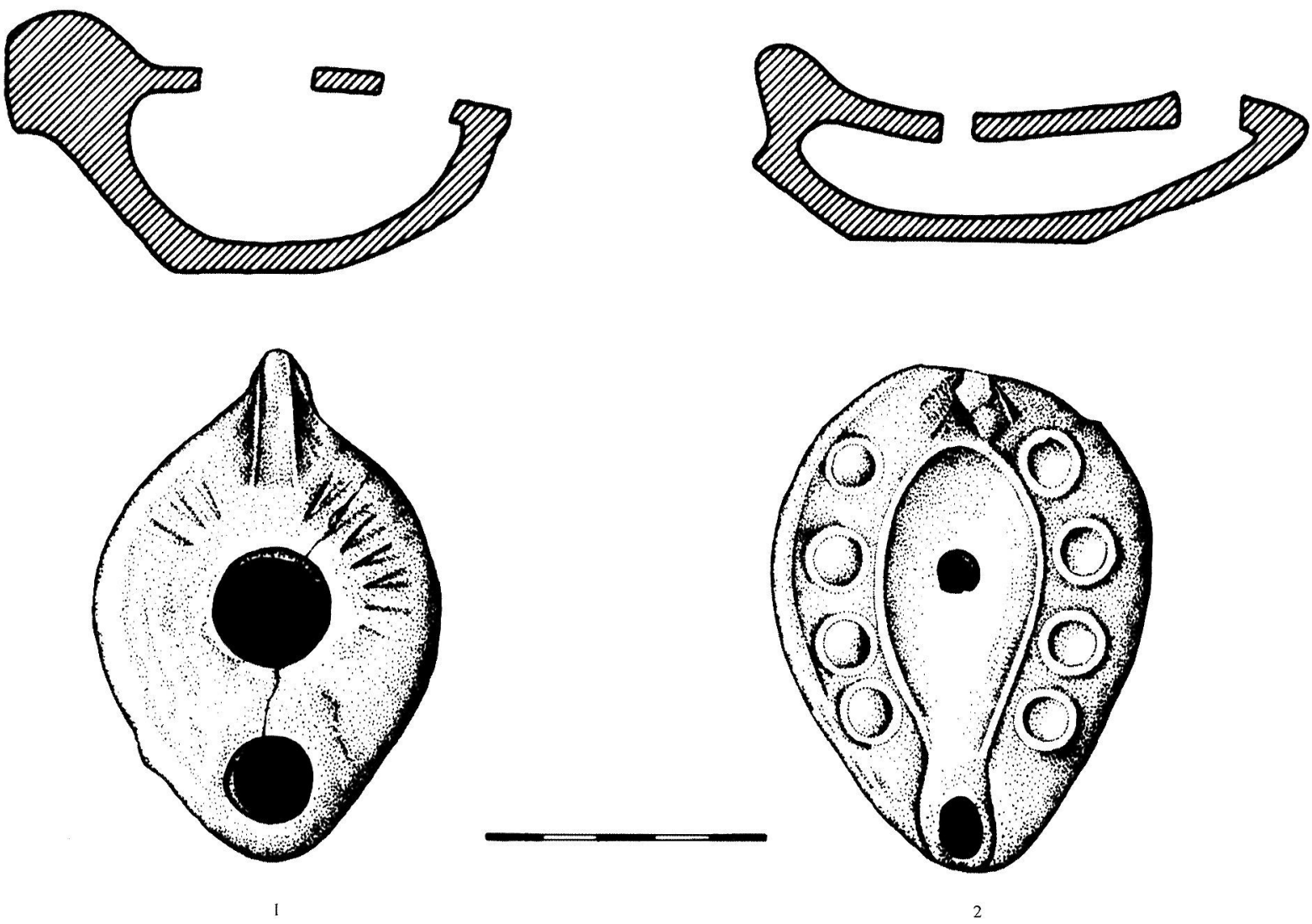

Figura 6

el rostrum se verifica mediante un canal. Cuenta con un asa maciza proyectada en vertical sobre el cuerpo de la lámpara.

Desde el punto de vista tipológico y temporal, la pieza se inscribe en los mismos parámetros que hemos explicitado para el ejemplar anterior. Algo similar sucede en lo que respecta al terreno decorativo, puesto que tampoco ha sido posible reconocer motivos idénticos en repertorios publicados, si bien es preciso advertir la semejanza existente entre el elemento floral que nos ocupa y el documentado sobre una lucerna del mismo tipo procedente de La Morlaca (AMARE, 1987, 31, Lám. III, núm. 10).

10.- Fig. 5,2.- Núm. Inv. 210. Procedencia: Huertas del Moro (Toledo), enero-mayo de 1899. Lucerna completa. Mantiene una longitudd máxima de $110 \mathrm{~mm} ., 71 \mathrm{~mm}$. de anchura y una altura de 59 $\mathrm{mm}$. desde el elemento de prehensión hasta la base. Superficie de tonalidad beige crema. Presenta cuerpo de forma oval sin distinción neta con el rostrum, ori- ficio de alimentación de importantes dimensiones (21 $\mathrm{mm}$.), y borde con decoración alterna de mamelones y líneas convergentes hacia el centro. La pieza está provista de un asa perforada.

Pieza de características morfológicas y decorativas análogas a las descritas han sido documentadas en Tamuda (PONSICH, 1961, Lám. XXX, núm. 459460), donde aparecen recogidas en el grupo destinado a albergar las lucernas de forma indeterminada. Esta circunstancia explica la ausencia en el citado conjunto mauritano, de referencia explícita a cuestiones relevantes tales como el área de fabricación y su cronología. Por lo que a nosotros respecta, el ejemplar toledano ha de incluirse sin ningún género de dudas en la categoría de lucernas romanas tardías, sin que sea posible establecer un marco temporal bien perfilado, dado el carácter descontextualizado del conjunto que sometemos a estudio.

11.- Fig. 6,1.- Núm. Inv. 211. Procedencia: Huerta del Rey Moro (Toledo). Lucerna completa. 
Ostenta una longitud máxima de $92 \mathrm{~mm}$. incluido el asa, $62 \mathrm{~mm}$. de ancho y una altura máxima de $47 \mathrm{~mm}$. desde el elemento de prehensión hasta la base. Superficie de tonalidad beige crema, muy desgastada y con evidencias de utilización en la piquera. Se trata de un ejemplar con infundibulum de forma ovoide, decorado con trazos radiales en la superficie anterior, provisto de orificio de alimentación central de importante diámetro $(20 \mathrm{~mm}$.) y asa maciza.

Una pieza idéntica a la que nos ocupa, forma parte del conjunto de lucernas conservado en el Museo de Sevilla (FENANDEZ CHICARRO, 1956, fig. 56,8); en la citada publicación, esta lámpara fue incluida en el grupo correspondiente a los especímenes de finales del siglo II y III d.C. A nuestro juicio, independientemente de la fecha sugerida por Fernández Chicarro, podría tratarse de una pieza de cronología algo más avanzada, a juzgar por la configuración general del objeto y su semejanza con una lucerna sin especificación morfológica explícita, incluida en la serie tardía del Museo del Louvre (HOFF, 1986, 155, núm. 281).

12.- Fig. 6,2. - Núm. Inv. 783. Procedencia concreta desconocida. Lucerna completa. Posee $98 \mathrm{~mm}$. de longitud máxima, una anchura de $68 \mathrm{~mm}$. y $35 \mathrm{~mm}$. de altura máxima, desde el elemento de prehensión hasta la base. Presenta superficie de coloración anaranjada, con restos evidentes de utilización en la piquera. Se trata de una pieza con infundibulum de desarrollo oval, disco de igual forma, prolongado hasta el rostrum a modo de canal y pequeño apéndice de prensión macizo que arranca en vertical desde el cuerpo del objeto. El borde aparece provisto de decoración consistente en cuatro círculos a cada lado.

Un ejemplar de idénticas características, procedente de Smirna, ha sido constatado en los fondos del Louvre (HOFF, 1986, 154, núm. 273). Piezas similares se hallan igualmente documentadas en la Colección Schloessinger, donde se le atribuye cronología bizantina (ROSENTHAL-SIVAN, 1978, núm. 586), así como en el Museo de Florencia (MICHELUCCI, 1975, 170, Lám. XXI, núm. 360).

No resulta tarea fácil adscribir nuestro ejemplar a una producción concreta, dado que la única pieza que conocemos que mantiene rasgos técnicos y decorativos idénticos a los de la lámpara del Museo de Santa Cruz, ha sido incluida en el Catálogo del Louvre en la categoría de «lucernas diversas y moldes». No obs- tante, sí puede afirmarse su relación formal con lámparas de origen sirio-palestino publicadas en el conjunto que venimos citando, datables entre los siglos $\mathrm{V}$ y VII d.C. (HOFF, 1978, 133 ss.), así como con otro grupo de características muy próximas, al parecer de fabricación egipcia y de similar cronología (MICHELUCCI, 1975, 105-106).

\section{CONSIDERACIONES FINALES}

El conjunto de lámparas romanas que hemos sometido a estudio, no permite, como resulta obvio, inferir una caracterización completa que posibilite el conocimiento exhaustivo de la lucernaria romana de Toledo y su provincia. Su propio carácter de objetos desprovistos de contexto arqueológico, así como el reducido volumen de ejemplares con que contamos, frenaría de modo decisivo toda tentativa encaminada hacia fines tan ambiciosos.

Tampoco ha de considerarse este trabajo como un catálogo completo de todos los objetos de esta índole que almacena el Museo, dado que no se incorporan a este análisis las piezas exhumadas en excavación. El objetivo básico propuesto no ha sido otro que el de dar a conocer al panorama científico la existencia de ejemplares, que de otro modo hubieran permanecido inéditos, al margen de una simple mención para alguno de ellos en el Catálogo del Museo (JORGE ARAGONESES, 1957, 62-63).

Como ya se indicó con anterioridad, el marco temporal reflejado por esta serie adquiere importante amplitud, tal y como habría de corresponder a un área en la que la presencia romana ha sido constatada arqueológicamente desde momentos tempranos, hasta las fases más tardías de la baja Romanidad. En efecto, la vieja Toletum aparece reflejada por vez primera en los textos clásicos por Livio $(35,22,5)$, con motivo de su toma por Fulvio en el 192 a.C. La documentación arqueológica atribuible a época republicana no es abundante en la propia ciudad, al tiempo que resulta aún sumamente parca a escala provincial (MENA, 1988, 30).

A medida que avanza el Imperio se incrementa de modo interesante el volumen de datos acerca de civitates, municipia y establecimientos de carácter rural acreditados en el marco territorial que constituye actualmente la provincia (RAMOS, 1988, 57). Por lo que se refiere a la fase tardorromana de Toletum, los fenómenos de cambio y transformación experi- 
mentados por la ciudad a partir del siglo IV d.C. comienzan a ser investigados en trabajos muy recientes (CARROBLES-RODRIGUEZ, 1988; CARROBLES, 1990).

Reflejo aproximado del devenir histórico que tan sucintamente hemos esbozado, resulta este lote de lucernas perteneciente al Museo de Santa Cruz. En efecto, contamos con el menos tres ejemplares adscribibles a época altoimperial. Especialmente destacables resultan, sin duda, los números 2 y 3 del catálogo, identificados como lucerna fabricada en Andújar y lucerna minera respectivamente. Ambas piezas presentan una interesante problemática específica que ha sido abordada en su momento oportuno por otros investigadores. Su documentación en Toledo, no hace sino engrosar los datos de dispersión disponibles hasta el presente, al tiempo que la primera de ellas, nos ha permitido replantear con mayores elementos de juicio algunas cuestiones en torno a la difusión de los productos generados por los talleres de origen.

La lucernaria romana tardía encuentra en la serie toledana que presentamos, una más que aceptable representación a través de ejemplares sumamente interesantes. La presencia de piezas de baja cronología en buena parte de los catálogos publicados constituye una constante, que no parece hallar reflejo en la existencia de estudios pormenorizados acerca de los puntos de origen y la datación aquilatada de las producciones. Salvo ciertas excepciones, buena parte de los paralelos constatados para nuestras piezas, han sido incluidos bajo el epígrafe de «formas diversas y moldes». Tal género de apartado suele funcionar a la manera de un «cajón de sastre» en el que se ubican todas aquellas lucernas que no han podido ser incluidas en otros grupos mejor caracterizados.

Dentro del conjunto toledano, destacaremos por su interés, las dos imitaciones de lucernas africanas que figuran en el catálogo con los números 8 y 9 . Se trata de piezas afines formalmente a la producción de Afrikanische Lampen, aunque con rasgos físicos y tecnológicos que inducen a pensar en un fenómeno de imitación, muy documentado por otra parte, en otros puntos del Imperio.

Desde el punto de vista de su localización espacial, resultan interesantes los ejemplares 10 y 11 , ingresados en el Museo a fines del pasado siglo, fruto de una serie de hallazgos producidos en la zona conocida como «Huertas del Rey Moro». Tan sugestivo topónimo se halla relacionado con la zona en la que hoy se ubica la estación de ferrocarril (JORGE ARAGONESES, 1957, 62). Merced a este tipo de materiales debe considerarse la existencia en este área de interesantes vestigios pertenecientes a la fase tardorromana de Toletum, que contribuirían a engrosar el volumen de conocimientos sobre el desarrollo de la ciudad en momentos tardíos.

\section{BIBLIOGRAFÍA}

ABASCAL, J.M., 1989: «Lucernas romanas de Segóbriga», Segóbriga III: La muralla Norte y la Puerta principal, Cuenca.

AMARE, T., 1984: «Nota sobre una lucerna de la Colección Gutiérrez Achútegui de Calahorra», Calahorra, Bimilenario de su fundación.

AMARE, T., 1987: Lucernas romanas de La Rioja, Logroño.

BAILEY, D.M., 1980: A catalogue of the Lamps in the British Museum. 2. Roman Lamps made in Italy, Londres.

BELCHIOR, C., 1969: Lucernas romanas de Conimbriga, Coimbra.

BLANCO GARCIA, F., 1987: Moneda y circulación monetaria en Coca. Siglo II a. C. a V d. C., Segovia.

BLANCO GARCIA, F., 1988: «Coca Arqueológica», Revista de Arqueología, 81, Madrid.

CABALLERO, L. y SANCHEZ PALENCIA, J., 1982: «Presas romanas y datos de poblamiento romano y medieval en la Provincia de Toledo», NAH, 14.

CARROBLES, J. y RODRIGUEZ, S., 1988: Memoria de las excavaciones de urgencia del solar del Nuevo Mercado de Abastos (Polígono Industrial, Toledo). Introducción al estudio de la ciudad de Toledo en el siglo IVd.C., Toledo.

CARROBLES, J., 1990: «Introducción a la Arqueología urbana en la ciudad de Toledo», I Congreso de Arqueología de la Provincia de Toledo, Toledo.

DAREMBERG, C. y SAGLIO, E., 1986: Dictionnaire des Antiquités grecques et romaines, Vol. III, 2, París.

DE CAROLIS, E., 1988: Lucerne Greche e Romane, Roma.

DELIBES, G. y MOURE, A., 1974: «Excavaciones arqueológicas en la villa romana de Almenara de Adaja (Provincia de Valladolid). Campaña de 1969», NAH, Arq. II, Madrid.

DeNeAUVE, J., 1969: Lampes de Carthague, París.

DOMERGUE, C., 1987: Catalogue des mines et des fonderies antiques de la Peninsule Iberique, Madrid.

FARINA, F., 1976: «Lucernas romanas de los Museos de Orense y Pontevedra», Boletin Auriense, VI, Orense.

FERNANDEZ CHICHARRO, C., 1956: «La colección de lucemas antiguas del Museo Arqueológico de Sevilla», Memorias de los Museos Arqueológicos Provinciales, XIII-XIV, 1952-53, Madrid.

FERNANDEZ GALIANO, D., 1984: Complutum I. Excavaciones, E.A.E., 137, Madrid.

FERNANDEZ OCHOA, C., 1980: «Notas sobre lucernas romanas de Asturias», Boletín del Instituto de Estudios Asturianos, 99, Oviedo.

FERNANDEZ OCHOA, C. y SELDA, I., 1987: «Lucernas romanas de La Bienvenida (Ciudad Real)», Oretum, III Ciudad 
Real.FERNANDEZ OCHOA, C., ZARZALEJOS, M. y SELDA, I., 1989: «Marcas de oficina en Terra Sigillata de la Submeseta Sur», XIX CNA, Zaragoza.

FERNANDEZ OCHOA, C. et alii, 1990: Sisapo I. Excavaciones en el yacimiento iberorromano de La Bienvenida (Almodóvar del Campo, Ciudad Real), en prensa.

FERNANDEZ OCHOA, C. y ZARZALEJOS, M., 1991 a): «Las producciones de Terra Sigillata altoimperial de Sisapo (La Bienvenida, Ciudad Real), Espacio, Tiempo y Forma, en prensa.

FERNANDEZ OCHOA, C. y ZARZALEJOS, M., 1991 b): «Terra sigillata del Museo de Ciudad Real. Colección Tello» Zephyrus, en prensa.

FERREIRA DE ALMEIDA, J.A., 1953: «Introduçao ao estudo das lucernas romanas em Portugal», O Arqueologo Portugues, $I I$.

GARABITO, T. et alii, 1986: «Los alfares romanos de Tricio y Arenzana de Arriba: Estado de la cuestión», II Coloquio de sobre Historia de la Rioja, 1985, Logroño.

GARCIA MERINO, C., 1990: «Uxama», Guía del Museo Numantino, Soria.

GIL FARRES, O., 1947-8: «Lucemas romanas decoradas del Museo Emeritense», Ampurias, IX-X.

HAYES, J.W., 1972: Late Roman Pottery, Londres.

HOFF, V., 1986: «Lampes romaines tardives et lampes chrétiennes en terre cuite», Musée du Louvre. Lampes en terre cuite grecques. Lampes en terre cuite chretiennes, París.

JORGE ARAGONESES, M., 1957: Museo Arqueológico de Toledo, Madrid.

LOESCHCKE, S., 1919: Lampen aus Vindonissa, Zurich.

LOPEZ RODRIGUEZ, J.R., 1981: «La colección de lucernas de la casa de la Condesa de Lebrija», BSAA, XLVII, Valladolid.

LOPEZ RODRIGUEZ, J.R., 1982: «Cuatro lucernas hispanorromanas», Homenaje a Conchita Fernández Chicarro, Sevilla.

LUZON, J.M., 1967: «Lucernas mineras de Riotinto», AEArq. XL. Madrid.

MANERA, E., 1960: Lucernas romanas de Pollentia en el Museo de Mallorca, Palma de Mallorca.

MENA, P., 1988: «La época republicana en Castilla-La Mancha: Inicios de la Romanización. (Siglo III-I a.C.)», I Congreso de Historia de Castilla-La Mancha, Vol. IV, Ciudad Real.

MENZEL, H., 1969: Antike Lampen im Römisch-Germanischen Zentralmuseum zu Mainz, Mainz.

MICHELUCCI, M., 1975: La collezione di lucerne del Museo Egizio di Firenze, Florencia.

MORILLO, A., 1991: «En torno a la tipología de lucernas romanas: problemas de nomenclatura», CuPAUAM, en prensa, Madrid.

MOUTINHO ALARÇAO, A. y DA PONTE, S., 1976: «Les lampes», Fouilles de Conimbriga IV, París.

PAVOLINI, C., 1977: «Le lucerne fittili del Museo Nazionale di Napoli», L'instrumentum domesticum di Ercolano e Pompei nella prima etá imperiale, Roma.

PÉREZ GONZALEZ, C., 1989: Cerámica romana de Herrera de Pisuerga (Palencia, Espana). La Terra Sigillata, Santiago de Chile.

POHL, G., 1962: «Die Frühchristhche Lampe von Lorenzberg bei Epfach, Landkreis Schongau. Versuch einer Gliederung der
Lampen von mediterraner Typus, aus Bayern Frühzeit», Schriften Bayer Landesgesh, 62, Munich.

PONSICH, M., 196I: Les lampes romaines en terre cuite de la Maurétanie Tingitane, Publications du Service des Antiquités du Maroc, Fasc. 15, Rabat.

PROVOOST, A., 1970: «Les lampes a recipient allongé trouvées dans les catacombes romaines. Essai de classification typologique», Bulletin de l'Institut Historique Belge de Rome, XLI, Roma.

QUINTERO ATAURI, P., 1932: Excavaciones en Cádiz, MJSEA, 117, Madrid.

RAMOS, J., 1988 «Romanización de Castilla La Mancha», I Congreso de Historia de Castílla-La Mancha, Vol. IV, Ciudad Real.

REYNOLDS, P., 1987: El yacimiento tardorromano de Lucentum (Benalua-Alicante): Las cerámicas finas, Alicante.

ROCA, M., 1980: «Sigillata importada y nuevas formas de Terra Sigillata Hispánica producidas en Andújar. Puntualizaciones cronológicas referidas a la actividad inicial del alfar», Cuadernos de Prehistoria de la Universidad de Granada, 5, Granada.

RODRÍGUEZ NEILA, J.F., 1977: «Serie de lucernas de Osuna», Habis, 8.

ROMERO, M.V., 1985: Numancia I. La terra sigillata, E.A.E., 146, Madrid.

ROSENTHAL, R. y SIVAN, R., 1978: Ancient Lamps in the Schloessinger Collection, Jerusalem.

SANCHEZ-LAFUENTE, J., 1990: Terra Sigillata de Segóbriga y ciudades del entorno: Valeria, Complutum y Ercávica, Madrid

SOLOVERA, E. y GARABITO, T., 1986: «Los nombres de los ceramistas romanos de La Rioja: Nuevas aportaciones», II Coloquio de Historia de La Rioja, 1985, Logroño.

SOLOVERA, E. y GARABITO, T., 1990: «Los talleres de Tritium Magallum. Nuevas aportaciones», Hispania Antiqua, Valladolid.

SOTOMAYOR, M. et alii, 1976: «Los alfares romanos de Andújar. Dos nuevas campañas», N.A.H., Arq. 4, Madrid.

SOTOMAYOR, M. et alii, 1979: «Los alfares romanos de Andújar. Campañas de 1974, 1975 y 1977», N.A.H., 6, Madrid.

SOTOMAYOR, M. et alii,, 1981: «Los alfares romanos de Los Villares de Andújar (Jaén, campaña 1978-1979)», N.A.H., 11, Madrid.

SOTOMAYOR, M. et alii, 1983: «Problemas de atribución en vertederos de TSH», Boletín del Museo Arqueológico Nacional, 2, Madrid.

SZENTLELEKY, T., 1969: Ancient Lamps, Chicago.

WALTERS, H.B., 1914: Catalogue of the Greek and Roman Lamps in the British Museum, Londres.

ZARZALEJOS, M., AURRECOECHEA, J. y FERNANDEZ OCHOA, C., 1988: «Amuletos fálicos romanos inéditos de las provincias de Madrid y Toledo", CUPAUAM, 15, Madrid

ZARZALEJOS, M., 1990: El yacimiento romano de Velilla de San Antonio (Madrid), Memoria de licenciatura inédita, UAM, Madrid. 